\title{
Polémiques toponymiques
}

La Ligue du Nord et l'usage politique du dialecte

Toponomical Polemics: the Northern League and the political use of dialects

\section{Martina Avanza}

\section{(2) OpenEdition}

Journals

Édition électronique

URL : http://journals.openedition.org/conflits/18054

DOI : $10.4000 /$ conflits. 18054

ISSN : $1777-5345$

Éditeur :

CCLS - Centre d'études sur les conflits lilberté et sécurité, L'Harmattan

Édition imprimée

Date de publication : 26 décembre 2010

Pagination : 123-138

ISBN : 978-2-296-56047-5

ISSN : 1157-996X

Référence électronique

Martina Avanza, "Polémiques toponymiques », Cultures \& Conflits [En ligne], 79-80 | Automne/Hiver 2010, mis en ligne le 26 mai 2012, consulté le 30 mars 2021. URL : http://journals.openedition.org/ conflits/18054; DOl : https://doi.org/10.4000/conflits. 18054 


\section{Polémiques toponymiques}

\section{La Ligue du Nord et I'usage politique du dialecte}

\section{Martina AVANZA}

Martina Avanza est enseignant chercheur à l'Université de Lausanne, IEPI (Institut d'études politiques et internationales) et co-directrice du CRAPUL (Centre de recherche sur l'action politique de l'Université de Lausanne). Ses travaux portent sur les partis politiques et le militantisme et privilégient une approche ethnographique de l'action politique. Elle a récemment publié: "The Northern League and its 'inoffensive' xenophobia", in Andrea Mammone et Giuseppe Veltri (dir.) Italy Today: The Sick Man of Europe, London, Routledge, 2010, pp. 131142.

T'équation « une langue, une nation » a largement fait ses preuves au XIXe _iècle ${ }^{1}$. Elle reste aujourd'hui encore très efficace, comme l'a montré, entre autres, l'éclatement de l'ex-Yougoslavie qui a conduit à une disjonction linguistique entre ses ressortissants. La Ligue du Nord ${ }^{2}$, parti qui s'emploie depuis 1995 à faire reconnaître la « Padanie » (à savoir, dans le langage partisan, les régions d'Italie septentrionale) comme une nation, ou tout au moins comme une entité culturellement distincte du reste du pays méritant pour cela d'être reconnue politiquement ${ }^{3}$, ne peut pas se prévaloir de cet argument. Les plus idéalistes des idéologues du parti ont essayé, un temps, de remédier à ce déficit de capital identitaire en tentant vainement de codifier une «langue padane » à partir des différents dialectes parlés dans les régions du nord ${ }^{4}$.

1. Thiesse A.-M., La création des identités nationales, Paris, Seuil, 1999.

2. Pour plus de détails sur le parti de la Ligue du Nord et son histoire, les deux ouvrages de référence sont : Biorcio R., La Padania Promessa. La storia, le idee e la logica d'azione della Lega Nord, Milan, Il Saggiatore 1997 et Diamanti I., La Lega. Geografia, storia e sociologia di un nuovo soggetto politico, Rome, Donzelli, 1993.

3. J'ai consacré ma thèse de doctorat à cette entreprise : Avanza M., Les "purs et durs de Padanie». Ethnographie du militantisme nationaliste à la Ligue du Nord, Italie (1999-2002), EHESS, 2007. Il s'agit d'une enquête ethnographique de longue durée parmi les militants léguistes les plus radicaux revendiquant l'indépendance de l'Italie septentrionale, rebaptisée Padanie.

4. Sur cette tentative voir Avanza M., «La Ligue du Nord : de la défense des dialectes à la recherche d'une langue nationale padane ", Mélanges de l'Ecole Française de Rome. Italie et Méditerranée, $\mathrm{n}^{\circ} 117 / 1,2005$, pp. 313-330. 
Comme le raconte Franco Rocchetta (l'un des fondateurs de la Ligue Vénète) : « quand j'ai connu Bossi 5 au début des années 1980, il avait sous le bras un tas d'imprimés avec lesquels il essayait de superposer les variables dialectales lombardes pour créer une langue unique ${ }^{6}$ ». Aujourd'hui, le professionnel chevronné de la politique qu'est Umberto Bossi a certainement oublié ses utopies linguistiques, mais la Ligue n'a pas pour autant abandonné l'idée de mener une politique de la langue. Pour combler l'absence d'une langue padane, le parti mise alors sur le «patrimoine linguistique padan » constitué par les dialectes septentrionaux que l'État aurait voulu sciemment détruire en imposant l'italien comme langue nationale ${ }^{7}$.

Cet article traitera d'un volet central de cette politique, à savoir des pratiques toponymiques de la Ligue qui consistent à diffuser sur les panneaux signalétiques, à côté du nom officiel en italien, le nom dialectal des villes et villages gouvernés par le parti. Dans cette pratique, « la fonction identitaire prend ostensiblement le pas sur la fonction de localisation : au delà de la nomination il s'agit d'un acte de nature nettement politique ou plus spécifiquement glottopolitique 8 ». Mais avant d'aborder la politique toponymique de la Ligue, et afin de comprendre les polémiques qu'elle soulève en Italie, il est d'abord nécessaire d'analyser la politisation des dialectes induite par l'usage qu'en fait la Ligue.

\section{L'appropriation des dialectes}

Depuis ses débuts, le parti a fait un usage politique du dialecte. C'est ainsi que Giuseppe Leoni, le tout premier élu léguiste (à Varèse, Lombardie, en 1985), a fait son entrée au conseil municipal en prononçant un discours en dialecte. L'initiative ayant été annoncée à la presse, le public s'est rendu nombreux à la session. Dans le récit qu'Umberto Bossi fait de l'événement, Leoni a commencé à lire son intervention dans un bruit assourdissant. Un homme s'est mis à applaudir Leoni en se moquant de lui, mais Bossi s'est levé énergiquement et l'a poussé dans les bras de la sécurité pour le faire sortir. Après cela, les opposants sont partis sans remous et Leoni a pu faire son intervention ${ }^{9}$.

5. Umberto Bossi a fondé la Ligue Lombarde en 1982 puis, en 1990, la Ligue du Nord qui absorbe les partis régionaux préexistants. Élu sans discontinuer (à la Chambre ou au Sénat) depuis 1987, il reste aujourd'hui le leader incontesté du parti.

6. Entretien accordé à Franco Rocchetta, Il Gazzettino, 15 mars 1993. Toutes les traductions de l'italien vers le français sont de l'auteur.

7. Pour la clarté du propos, nous reprenons la distinction langue (l'italien) et dialectes (parlers locaux) tout en sachant qu'il s'agit d'une séparation qui ne va pas de soi ni linguistiquement ni politiquement. Les léguistes, en effet, refusent cette séparation et préfèrent parler de "langues locales ", le terme " dialecte » étant perçu comme dévalorisant. Pour appuyer leur thèse, ils citent souvent Noam Chomsky pour lequel les dialectes sont des langues comme les autres, sauf qu'elles sont dépourvues d'une armée. En réalité, la citation exacte est « une langue est un dialecte avec une armée » et, si Chomsky l'utilise souvent, il l'attribue au linguiste yiddish Max Weinreich. 
Un tel usage du dialecte représente en Italie une véritable rupture. Alors que l'utilisation du dialecte est courante en famille ${ }^{10}$, cet usage est considéré comme totalement iconoclaste non seulement dans le champ politique, mais aussi plus généralement dans l'espace public. En effet, si l'on excepte un usage artistique (essentiellement poésie, chanson et comédies dialectales) marginal mais réel, le dialecte est proscrit à l'école, dans les administrations ou dans les médias. La bonne maittrise de l'italien et l'absence d'un fort accent local correspondant encore, dans l'Italie septentrionale, à un signe de distinction sociale, l'utilisation du dialecte dans l'espace public y est vue comme l'aveu d'une incapacité, jamais comme un choix. Celui qui parle dialecte est donc souvent catalogué comme rustre, ignorant, provincial, surtout lorsqu'il s'agit des parlers gutturaux des petites villes et villages de l'aire pré-alpine, celle précisément qui constitue le terroir léguiste. Ici, encore plus qu'ailleurs, la mâ̂trise du code linguistique, de ses normes et de ses critères de correction, sont des indicateurs du capital culturel des locuteurs ${ }^{11}$. Ainsi, parler bergamasque, Bergame étant un fief léguiste, est une marque d'analphabétisme. De même, si l'accent milanais est «chic» (puisque associé à une grande ville, capitale économique du pays), celui de Varèse (ville d'origine d'Umberto Bossi) est «moche et ridicule », y compris pour une bonne partie de ses locuteurs qui ont intériorisé ces jugements esthétiques et sociaux.

Selon un schéma bien connu ${ }^{12}$, le parti renverse ce stigmate en attribuant une valeur positive aux indices infamants du provincialisme linguistique. Le fait d'avoir un accent ou un dialecte connotés négativement ne représente pas, aux yeux des léguistes, une incapacité à participer à l'élite culturelle et à ses codes, mais un choix d' 'identité 13 linguistique ». L'accent et le dialecte (les deux étant liés) les plus généralement méprisés sont alors exhibés avec fierté. La Ligue ne se contente pas de cet usage symbolique et provocateur du dia-

8. Boyer H., «Fonctionnements sociolinguistiques de la dénomination toponymique ", Mots. Les langages du politique, $\mathrm{n}^{\circ} 86,2008, \mathrm{p} .11$.

9. Bossi U., La Lega 1970-1989, Milan, Editoriale Nord, 1999, pp. 63-64.

10. Au moment de l'unité (1861), la proportion d'italianophones est estimée à $2,5 \%$ de la population, dont la plupart étaient des Toscans ou des Romains. L'Italie est d'ailleurs restée essentiellement dialectophone jusqu'au lendemain de la deuxième guerre mondiale. Voir De Mauro T., Storia linguistica dell'Italia unita, Bari, Laterza, 2003. De plus, être en mesure de maîtriser l'italien ne signifiait pas pour autant l'utiliser. Selon l'institut national des statistiques (ISTAT), en 2000, si $58 \%$ des Lombards et $22 \%$ des Vénètes parlent presque exclusivement l'italien en famille, $10 \%$ des Lombards et $42 \%$ des Vénètes parlent presque exclusivement dialecte et $28 \%$ et $30 \%$ respectivement en font un usage mixte. Par rapport aux années 1980 et 1990, l'ISTAT constate que l'utilisation exclusive de l'italien se consolide et que l'utilisation exclusive du dialecte diminue, mais aussi que l'utilisation mixte italien/dialecte, qui augmente de manière significative, compense en partie cette baisse.

11. Bourdieu P., Ce que parler vent dire, Paris, Fayard, 1982.

12. Goffman E., Stigmate, Paris, Minuit, 1975.

13. Le mot « identité » est mis entre guillemets dans cet article car il s'agit pour nous d'un terme indigène (un concept utilisé par les acteurs) et non d'un terme scientifique ayant une portée heuristique. Sur cette question voir : Brubaker R., "Au-delà de l'identité », Actes de la recherche en sciences sociales, $\mathrm{n}^{\circ}$ 139, 2001, pp. 66-85 et Avanza M. et Laferté G., « Dépasser la "construction des identités" ? Identification, image sociale, appartenance ", Genèses, n 61, 2005, pp. 154-167. 
lecte, elle tente aussi de promouvoir des politiques publiques visant à lui donner un statut juridique. Au niveau national, le parti a essayé de tirer profit de la «Charte européenne des langues régionales et minoritaires». En guise de ratification de cette Charte, le Parlement italien a voté une loi sur les minorités ethniques et linguistiques (la loi n 3366 du 25 novembre 1999). Cette dernière concerne les petites minorités francophones, germanophones, albanaises, ou encore slovènes du pays, mais ne reconnaît que le frioulan et le sarde parmi les langues régionales (la Sardaigne et le Frioul sont des régions à statut extraordinaire ${ }^{14}$ ). Etant considérés comme des dialectes et non comme des langues, les parlers régionaux des régions ordinaires (mais aussi de certaines régions à statut spécial comme la Sicile) n'ont pas été pris en compte. Cette situation, perçue comme un manque de reconnaissance totalement injustifié, fournit à la Ligue une occasion d'exprimer des propos xénophobes. Ainsi, Gilberto Oneto (à l'époque principal idéologue du parti) s'est insurgé dans le quotidien partisan, La Padania, contre le fait que la langue des "gitans voleurs », le rom, soit considéré, dans le projet de loi, comme une langue à protéger, contrairement aux parlers du Nord. Il se demandait ensuite, outré, si l'État italien voulait également s'occuper des droits linguistiques des Marocains, des Albanais et des Sénégalais (qui constituaient à l'époque la majorité de la population immigrée) ${ }^{15}$. Entre la défense de «l'identité » et la xénophobie, au sein de la Ligue, la frontière est toujours poreuse. Roberto Calderoli, alors ministre, a ainsi présenté en novembre 2003 un projet de loi, resté lettre morte, pour que les étrangers demandant la nationalité italienne soient soumis non seulement à un test d'italien, mais aussi à une épreuve de «langue locale » de leur lieu de résidence. De tels usages du dialecte contribuent à sa politisation en faisant des parlers locaux la marque d'une attitude fermée, voire xénophobe.

$\mathrm{Si}$, au niveau du Parlement, la Ligue se révèle incapable de promouvoir des politiques publiques en faveur des dialectes (sauf dans le domaine de la toponymie, comme on le verra plus loin), le parti a eu plus de succès localement, au sein des conseils régionaux, provinciaux et municipaux. Dans le cadre d'une politique de décentralisation de plus en plus poussée, le niveau régional est particulièrement important. Pour pouvoir agir à ce niveau, la Ligue a institué suite aux élections régionales de 2000, dans les régions de Lombardie, Vénétie et Frioul (gouvernées par la coalition de droite à laquelle la Ligue appartient), des mandats d'adjoint aux «cultures, identités et autonomies » (auparavant on parlait simplement d'adjoint à la culture) et a imposé à ces

14. Il s'agit de régions frontalières et insulaires, souvent marquées par des particularismes linguistiques, qui, dès l'après-guerre, avaient obtenu une large autonomie tant au niveau culturel et linguistique (allant jusqu'au bilinguisme pour le Val d'Aoste et le Trentin) qu'aux niveaux politique et économique. La Constitution (titre V, art 114 et 116) différencie donc des « régions à statut spécial » (Sicile, Sardaigne, Frioul, Val d'Aoste et Trentin) et des « régions ordinaires » (toutes les autres). La Ligue s'est implantée dans ces dernières et n’a jamais percé (à l'exception du Frioul) dans les régions à statut spécial.

15. Oneto G., « La Padania é sacra », La Padania, 29 juin 1998. 
postes des léguistes très attentifs au thème du dialecte et plus généralement aux thématiques identitaires chères au parti. Arrêtons-nous sur le cas de la Lombardie, d'où seront tirés les exemples de polémiques toponymiques dont nous traiterons dans la suite de l'article. Dans cette région, entre 2000 et 2005 le poste d'adjoint aux «cultures, identités et autonomies » était occupé par le professeur universitaire Ettore Albertoni. Ce dernier a ouvert en juin 2001 un «Centre de documentation et de coordination des études sur la Lombardie » entièrement consacré aux dialectes. Il a en outre placé à la direction du centre un léguiste de la première heure responsable du secteur culturel au sein $\mathrm{du}$ parti. Pendant son mandat, Albertoni a également fait éditer un lexique lombard 16 et une grammaire des dialectes lombards ${ }^{17}$. Suite aux élections régionales de 2005, gagnées à nouveau par la coalition de droite, Albertoni, devenu président du Conseil régional, cède la place à un autre léguiste, Massimo Zanello, qui l'a occupée jusqu'en 2010. Tout en continuant à financer le «Centre de documentation et de coordination des études sur la Lombardie », ce dernier semble moins attentif à la thématique dialectale que son prédécesseur. Il reste que la Lombardie (en profitant du fait que l'Italie a ratifié en 2007 la convention de l'UNESCO sur la protection de la culture immatérielle) a voté une loi régionale de valorisation du patrimoine culturel immatériel dont les principes d'application précisent qu'elle concerne aussi les dialectes et la toponymie (loi régionale $\mathrm{n}^{\circ} 27 \mathrm{du} 23$ octobre 2008). Des initiatives du même type, mais de moindre envergure, sont également financées par les provinces et les communes où la Ligue gouverne ou participe au gouvernement local. Le volet toponymique de ces politiques régionales, provinciales ou municipales sera détaillé plus loin.

À côté des élus, les médias du parti sont également mis à contribution de la cause dialectale. La Padania, quotidien partisan, loue régulièrement les actions entreprises par la Ligue pour défendre les dialectes. Le journal relate aussi les activités organisées par les associations des amateurs du dialecte, y compris quand celles-ci ne sont pas liées au parti, et fait la promotion de comédies en dialecte, de concerts de chanson dialectale. Le quotidien prend même des initiatives propres comme le concours de poésie dialectale qu'il lance auprès de ses lecteurs et dont le gagnant a été primé, en février 2010, par le ministre Umberto Bossi en personne, qui a lui-même écrit de la poésie dialectale dans sa jeunesse ${ }^{18}$. Dans Quaderni Padani, revue culturelle indépendante mais proche du parti, le débat sur le dialecte, plus pointu, a une place fondamentale : on y trouve de nombreuses études de linguistique et de toponymie. La radio du mouvement (Radio Padania Libera) organise des cours de dialecte, incite les auditeurs à envoyer des poésies dans leur « langue identi-

16. Beretta C. (ed.), Parlate e dialetti della Lombardia. Lessico comparato, Milan, Mondadori, 2003.

17. Rognoni A. (ed.), Grammatica dei dialetti della Lombardia, Milan, Mondadori, 2005

18. Sur Bossi poète voir Avanza M., «Une politique qui vole sur les ailes de la poésie. Pratiques politico-poétiques au sein de la Ligue du Nord », Terrain, n 41, 2003, pp. 47-62. 
taire » et diffuse très souvent des chansons dialectales (ce qui n'est pas du tout le cas des autres radios de la région). L'association Internet Padano, animée par des léguistes, contribue à cette valorisation du dialecte et de ses défenseurs : elle répertorie les sites sur les dialectes, très nombreux, sans se soucier de leur orientation politique ou les héberge gratuitement dans sa communauté virtuelle Padaniacity (www.Padaniacity.org), au même titre que d'autres sites considérés comme «identitaires ».

Tout comme les médias, l'organisation partisane contribue à la visibilisation de la cause dialectale. Ainsi, les associations padanes (associations promues par le parti et s'occupant de thèmes aussi variés que l'art, le sport ou la jeunesse) organisent des cours de dialecte, des conférences sur la littérature en dialecte et des concours de poésie dialectale. Les sections locales du parti sont également mobilisées. À titre d'exemple, à Bergame, le parti a organisé en janvier 2003 un colloque intitulé "La renaissance des langues locales », dans lequel est intervenu, entre autres, Ettore Albertoni. En menant la bataille au Parlement autour de la Charte européenne des langues minoritaires et régionales, en promouvant au niveau local, provincial ou régional des initiatives pour la protection des dialectes et en mobilisant ses réseaux et médias militants autour de ces thèmes, la Ligue se présente comme le seul acteur politique s'occupant du dialecte et de sa défense. De cette manière, elle récupère la cause dialectale et la fait coïncider avec le parti, alors qu'une grande partie des associations réunissant les amateurs du dialecte ne sont ni liées à la Ligue, ni en accord avec son projet indépendantiste ou autonomiste et sa politique xénophobe.

Ce monopole de la Ligue dans l'utilisation politique du dialecte est relativement récent. Umberto Bossi raconte qu'à la fin des années 1970, et alors qu'il se demandait sur quels arguments fonder un mouvement autonomiste lombard, il n'avait pas pu se légitimer en utilisant le dialecte. En effet, à cette époque, le parti communiste défendait les dialectes en tant qu'expressions populaires et antifascistes ${ }^{19}$. A présent, les partis héritiers du parti communiste ne se soucient plus de ces questions et la Ligue peut alors endosser le rôle de défenseur de "l’identité linguistique » du Nord. Certains élus de gauche semblent aujourd'hui regretter d'avoir " permis à la Ligue de s'être appropriée la bataille pour la valorisation et la défense de la culture locale et dialectale », mais se rendent compte qu'il est désormais trop tard : « ...il aurait fallu intervenir avant. On aurait pu mettre de côté un certain snobisme typique de la gauche et reconnaître que les traditions (les vraies) et le dialecte, y compris celui de Bergame, même s'il est dur et cacophonique, doivent être préservés et valorisés et qu'il s'agit d'une bataille culturelle qui ne peut être abandonnée à un seul parti $20 »$.

19. Bossi U., Il moi progetto : discorsi su Federalismo e Padania, Milan, Sperling \& Kupfer, 1996, pp. 19-20. 
La Ligue monopolisant, au Nord, la question dialectale, toute utilisation publique du dialecte ou toute action volontariste est automatiquement associée au léguisme. Le dialecte s'est alors transformé, dans son utilisation publique, en une marque de fermeture, voire de xénophobie, et d'une attitude anti-unitaire. Umberto Bossi exprime d'ailleurs clairement les raisons pour lesquelles la Ligue s'est intéressée au dialecte et aux formes d'expression associées : «la chanson et la poésie dialectales... même si elles n'ont jamais été codifiées de la sorte, ... sont un sublime acte d'accusation contre les patrons italiens, les intellectuels italiens, les policiers italiens 21 ». Marqueur d'autochtonie, le dialecte serait anti-italien et donc implicitement padan. Cette hostilité envers l'État italien se double d'une attitude de rejet envers les Italiens du Sud, notamment ceux qui, nombreux, ont migré au Nord. Mettre en avant le dialecte est pour la Ligue, une façon de souligner que ces derniers ne sont pas tout à fait chez eux, tout comme les immigrés. Cette politisation, à droite, du dialecte sera illustrée ici à travers le cas des panneaux signalétiques bilingues (italien/dialecte) que de nombreux maires léguistes ont voulu installer et par les polémiques que cette action a suscitées.

\section{L'enjeu toponymique}

A partir du virage indépendantiste de la Ligue du Nord (en 1995, de fédéraliste la Ligue devient officiellement sécessionniste), on a assisté à une idéologisation et à une radicalisation de la fonction d'élu local qui, d'administrateur, est sommé de devenir avant-garde de la lutte de «libération nationale». Il s'agit de traduire la revendication padane au niveau local à travers l'organisation d'activités culturelles : théâtre dialectal, réécriture, en version padane, de l'histoire locale, musique celtique ${ }^{22}$. Le versant principal de cette politique concerne le marquage du territoire qui se traduit par l'utilisation de symboles partisans dans des lieux publics (des "Soleil des Alpes », symbole figurant sur le drapeau de la future nation padane, apparaissent sur les bâtiments publics ${ }^{23}$ ), par la pratique consistant à changer les noms des rues en les " padanisant » (la rue "Rome" devient ainsi rue "Padanie") et par le fait de traduire en dialecte les noms de villes et villages. Dans cet article, nous développerons

20. http://stefanozenoni.splinder.com, post du 11 avril 2009. L'auteur du blog est élu au conseil communal de Bergame dans les rangs de l'opposition de gauche. Un autre exemple (ils sont rares) est la rencontre organisée en mai 2002 à Chivasso (province de Turin) par Refondation Communiste (parti d'extrême gauche) intitulée « La Langue piémontaise : une ressource culturelle européenne que la gauche ne doit pas laisser étouffer entre la Ligue et la globalisation".

21. Bossi U. et Vimercati D., Processo alla Lega, Milan, Sperling \& Kupfer, 1998, p. 26.

22. Les padanistes se pensent comme des descendants des Celtes, contre les méridionaux latins.

23. Ainsi le maire léguiste de Adro (province de Brescia) a fait décorer la toute nouvelle école publique inaugurée en septembre 2010 avec des centaines de "soleil des Alpes ». Les léguistes soutiennent que ce symbole, qui figure pourtant non seulement sur le drapeau « padan » mais aussi sur le symbole électoral de la Ligue, n'est pas un signe partisan, mais un symbole celtique témoignant donc des " traditions padanes ". Pour des photos voir http://www.repubblica.it/cronaca/2010/09/18/foto/adro_la_scuola_con_i_simboli_della_leg a-7203499/1/ 
ce dernier aspect. Cependant la question de la toponymie dialectale doit être comprise dans un cadre plus large de padanisation du territoire. En inscrivant la Padanie dans la toponymie, la géographie et les bâtiments publics, les élus locaux léguistes imposent « leur » nation même à ceux qui ne s'y reconnaissent pas, ce qui suscite évidemment des conflits.

Durant la phase indépendantiste, ces pratiques étaient ouvertement encouragées par le parti. Les élus locaux léguistes étaient encadrés par des « responsables des collectivités territoriales » lorsqu'ils se trouvaient dans l'opposition et par le Padania Office pour les maires et les conseillers de la majorité. Elena a travaillé dans ces deux structures en pleine phase indépendantiste. Son rôle était d'organiser des réunions avec les élus de la Brianza (zone au Nord de Milan), dont six maires, afin d'homogénéiser leurs pratiques et de leur conférer une orientation " padaniste » : « à cette époque, on insistait beaucoup sur l'indépendance. Alors il fallait leur expliquer comment faire des rues consacrées à ... (inaudible), comment faire les panneaux en langue locale. Il fallait leur dire qu'ils devaient tous aller au conseil municipal avec la pochette verte (la couleur du parti), même des trucs bêtes, mais c'était important qu'il y ait un symbole 24 ». Même s'ils ne sont plus systématiquement poussés dans ce sens, les élus léguistes ont souvent continué à mettre en place une politique de padanisation du territoire bien après que le parti ait renoncé officiellement à l'indépendance (en 2000). La Ligue regarde avec sympathie ces pratiques qui ne sont en aucun cas sanctionnées, voire qui continuent d'être encouragées. Ainsi, lors d'une session de l'école politique fédérale, en octobre 2003, alors que la Ligue gouverne le pays avec ses alliés de droite, les élus locaux étaient encore invités à intituler des rues ou des places à la Padanie ou au «Soleil des Alpes », façon de concrétiser au niveau local la «tutelle et la valorisation de l'identité » 25 .

Le fait de pouvoir ajouter le nom dialectal au nom officiel des villes et villages est une vieille revendication des partis autonomistes. Ce droit a été concédé dans l'après-guerre, dans les régions à statut spécial, aux minorités francophones, germanophones et slavophones. Les partis autonomistes des régions ordinaires ont alors demandé l'extension de ce droit aux dialectes, mais ils ne l'ont jamais obtenue. La Ligue a repris à son compte cette demande qui, dans le cadre d'une revendication indépendantiste, a pris une tournure beaucoup plus radicale : pour un maire léguiste, rebaptiser la ville avec son nom dialectal, c'est un peu quitter l'État italien et s'affranchir de sa langue. Le Ministère de l'intérieur ne s'y est d'ailleurs pas trompé : en mars 1996 (six mois avant que la Ligue déclare, symboliquement, l'indépendance de la Padanie lors d'une grande manifestation), il promulguait une circulaire concernant la toponymie $\left(n^{\circ} 4 / 96\right)$. Cette circulaire signalait aux collectivités

24. Entretien avec Elena, Milan, siège de la Ligue, avril 2002.

25. Maria Piera Pastore, "Struttura e funzioni degli enti locali », Scuola politica federale, Boveno, 4-5 octobre, 2003. Document partisan. 
locales qu'elles devaient «se tenir aux dispositions qui prévoient le bilinguisme uniquement là où il est effectivement en vigueur, ce qui exclut l'utilisation du dialecte, qui, en l'état actuel de la législation, ne jouit d'aucune tutelle en ce sens ». Gilberto Oneto, considérant cette circulaire comme le signe d'une véritable oppression, rappelait que la norme en vigueur était une loi fasciste promulguée en juin 1927 26. Dénonçant « l'italianisation stupide et offensive » subie par la toponymie padane 27 mise en ouvre par les « militaires de l'IGM 28 » qui «se sont comportés comme les fonctionnaires de toutes les puissances coloniales », Oneto invitait les élus locaux à la « résistance ». Ils devaient ignorer la circulaire et continuer à rajouter son « vrai » nom au nom italien de la commune. Pour aider les élus dans cette opération, Quaderni Padani, revue « savante » du padanisme dirigée par Oneto lui-même, a commencé à publier, à partir du numéro 6 sorti en 1996, la liste des « véritables noms de nos villes et villages». Le numéro 13, sorti en 1997 et entièrement consacré à la toponymie, reprend l'intégralité de cette liste contenant « tous les noms vernaculaires des communes padanes ». Il s'agit de la transcription d'appellations dialectales qui, pour la plupart, n'existent que sous une forme orale. Oneto est conscient qu'il peut y avoir des fautes de transcription (pour lesquelles les amateurs du dialecte l'ont d'ailleurs fortement critiqué), mais, malgré ces imperfections, il pense qu'il était urgent d'avoir cette liste afin que les élus locaux puissent s'en servir. Pour leur politique toponymique, ces derniers disposent depuis peu d'un outil plus officiel que la liste publiée dans Quaderni Padani. En effet, le "Centre de documentation et de coordination des études sur la Lombardie » a publié en 2010 un ouvrage entièrement consacré à la question ${ }^{29}$. De nombreux élus léguistes semblent s'être servis de la liste d'Oneto. Ainsi, en 2009, la région Lombardie estimait que la toponymie bilingue dialecte-italien avait été adoptée dans 267 communes 30 sur un total de 1546 (plus d'une commune sur six donc). La région ne précise pas quels partis gouvernaient ces communes, mais nous pouvons raisonnablement supposer que ces initiatives sont le fait de la Ligue. En tout cas, nous n'avons eu connaissance d'aucun cas de signalétique bilingue voulue par des élus d'un autre parti.

Le phénomène des panneaux bilingues a concerné surtout les petits centres, là où la Ligue est électoralement le mieux implantée, mais aussi quelques chefs-lieux de province comme Bergame (Lombardie), cas sur lequel nous reviendrons plus longuement. Parfois, comme à Varèse (chef-lieu de province en Lombardie) et à Cantù (Lombardie), les maires ou leurs adjoints à la cul-

26. Oneto G., «L'oppressione toponomastica italiana », Quaderni Padani, n 13, 1997.

27. Il cite à ce titre des exemples. Au Piémont, Ser vej (hauteur ancienne) est devenu Cervelli (cerveaux) et Mj rotond (lacs ronds) Mogli Rotonde (épouses rondes). En Lombardie, Senar (cendres, lieu des cendres) s'est transformé en San Re (Saint roi) et il y a encore "plein d'autres exemples idiots».

28. Istituto Geografico Militare qui a en charge le service cartographique du pays.

29. Rognoni A., Toponomastica della Lombardia, Mursia 2010.

30. Il Corriere della Sera, 27 février 2009. 
ture ont réuni des commissions d'étude pour retrouver les « noms historiques » des rues datant d'avant «l'italianisation ». Ils ont donc changé non pas le nom de la ville, mais les plaques des rues, comportant désormais le nom officiel et le nom « local ». Ainsi, à Cantù, en 2001, au Largo Adua a été associé Pianela, "écriteau en lombard rapportant la dénomination du début du XX siècle, la dénomination historique liée aux usages populaires 31 ». À Varèse, en 2000, le maire léguiste a voulu jouer la carte de la modernité. Il n’a pas changé les plaques de rues, mais a fait rajouter des panneaux électriques lumineux indiquant le nom de lieux dits ou de bâtiments historiques en dialecte et en anglais. Pour sortir ses maires de l'illégalité qui caractérisaient de telles opérations, la Ligue avait inséré la question de la toponymie dans le projet de loi constitutionnelle sur la dévolution (novembre 2001) 32. Il s'agissait d'une importante transformation impliquant de modifier la Constitution (art. 117) pour décentraliser vers les régions de vastes pouvoirs (la police, l'éducation, la santé) qu'elles exerceraient de manière exclusive. Ce projet faisait de la toponymie une matière de compétence exclusive des régions, ce qui aurait de fait accru le pouvoir de la Ligue en la matière (le parti et ses alliés gouvernant presque toutes les régions septentrionales). Luciano Vandelli ${ }^{33}$, en analysant le parcours législatif de la loi sur la dévolution, s'étonne d'une telle précision, puisque la question de la toponymie lui semble sans importance dans le cadre d'une réforme institutionnelle de grande envergure. Mais cette matière est emblématique : réintroduire le «vrai » nom des villages est pour les padanistes une façon de dire qu'ils sont chez eux et que chez eux ce n'est pas l'Italie. On peut d'autant plus comprendre que le projet de loi traite de la toponymie, quand on sait que c'est le parlementaire européen Francesco Speroni qui est directement intervenu en ce sens. Or, à cette époque, Speroni présidait le Parlement Padan (Parlement virtuel animé une fois par mois par les militants) dont la commission culture (le Parlement Padan était divisé en commissions thématiques) avait rédigé un projet de loi visant à récupérer la « toponymie historique ». Speroni, comme il le dit lui-même, se fait alors le porte-parole du Parlement Padan en faisant remonter des propositions auxquelles les militants sont attachés ${ }^{34}$.

Si la Dévolution, votée au Parlement en 2005, a été abrogée par référendum en 2006 (et n'est donc jamais devenue effective), la Ligue a néanmoins réussi à trouver un moyen pour légiférer sur la toponymie. Profitant du vote du nouveau code de la route, le parti, alors au gouvernement, a proposé un

31. Corriere di Como, 13 novembre 2001.

32. En 2000, la Ligue renonce à demander l'indépendance de la Padanie afin de renouer avec ses alliés de droite (Berlusconi et Alliance Nationale) qui refusaient de traiter avec un parti sécessionniste. Mais le parti obtient de ses alliés qu'ils soutiennent une transformation fédérale du pays appelée dévolution en écho au modèle écossais. La coalition de ces trois partis ayant gagné les élections législatives de 2001, la Ligue s'attelle donc à promouvoir son projet.

33. Vandelli L., Devolution e altre storie. Paradossi, ambiguità e rischi di un progetto politico, Bologne, Il Mulino, 2002.

34. Lire l'interview de Francesco Speroni dans La Padania, 22 novembre 2001. 
amendement introduisant la possibilité de rajouter le nom en dialecte sur les panneaux signalétiques touristiques de compétence des communes (se distinguant des autres par leur couleur marron). Inséré dans un texte technique (il s'agissait d'introduire le permis à point et autres mesures du même ordre), cet amendement est passé inaperçu, ce qui aurait été différent dans le cas d'une loi portant spécifiquement sur la langue, matière plus symbolique et pour cela délicate. Le nouveau code de la route ayant été approuvé le 27 juin 2003, les léguistes ont donc fini par obtenir le pouvoir d'utiliser le dialecte dans la signalétique. Cela dit, comme le montre le cas controversé de Bergame, la légalité des panneaux n'est pas une garantie de leur pérennité.

\section{Quand Bergamo devient Berghèm... et vice versa.}

Bergame et sa province sont l'un des bastions de la Ligue. Le maire de la ville n'a pourtant jamais été léguiste. Comme cela arrive souvent là où le parti est suffisamment fort pour avoir des élus en se présentant seul, la Ligue, jusqu'en juin 2009, n'a jamais voulu s'allier aux partis de droite pour les élections municipales, y compris lorsqu'elle gouvernait le pays avec ces mêmes partis (comme en 2004). En 1995 et en 2004, cette dispersion des forces de droite a permis à la coalition de centre-gauche, pourtant minoritaire dans la ville, de gagner les municipales. En 1999, en revanche, Cesare Veneziani, soutenu par Forza Italia, Alliance Nationale et UDC (catholiques) l'a emporté malgré la présence d'un candidat léguiste. La Ligue, à l'époque encore indépendantiste, s'est donc retrouvée dans l'opposition au conseil municipal. Mais, après les élections législatives de 2001, la Ligue a réintégré la coalition de droite. Ce contexte national a eu des répercussions locales. Le maire Cesare Veneziani se devait désormais de prendre davantage en considération les demandes des conseillers léguistes qui, en échange et s'ils étaient restés officiellement dans l'opposition, se devaient de ne pas être trop critiques envers le maire. La Ligue a profité de cet échange de bons procédés pour faire voter en avril 2002 l'une de ses vieilles revendications, l'installation de panneaux dialectaux. La motion léguiste proposait d'ajouter à la signalétique officielle des panneaux touristiques (de couleur marron) portant l'inscription «Berghèm » pour les installer à l'entrée de la ville. Des tentatives en ce sens avaient été faites depuis 1995 par les conseillers léguistes, et notamment par le secrétaire de la Ligue à Bergame, le jeune comptable Daniele Belotti, mais la précédente majorité de centre-gauche n'avait jamais voulu en entendre parler. En 2002, en revanche, la motion léguiste a été approuvée grâce aux votes des conseillers de la Ligue, mais aussi de Forza Italia (le parti de Silvio Berlusconi). L'opposition de gauche a voté contre, tout comme le groupe d'Alliance Nationale (droite de descendance fasciste et pour cela attachée à l'unité nationale ${ }^{35}$ ) qui faisait partie de la majorité municipale. Constatant le désaccord de

35. Malgré le fait que la Ligue et AN aient longtemps fait partie de la même coalition de droite (coalition dirigée par Silvio Berlusconi qui a gouverné le pays entre 1994-95, 2001-2006 et depuis 2008), les deux partis se sont opposés sur la question de l'unité nationale que AN a 
sa majorité sur la question, le maire de Forza Italia n'a pas voté avec les conseillers de son groupe et s'est abstenu. Bergame est ainsi devenue le premier chef-lieu de province (plus de 130.000 habitants) à avoir son nom en dialecte à l'entrée de la ville, ce qui n'est pas passé inaperçu.

Le ministre des infrastructures Pietro Lunardi, de Forza Italia, appartenant pourtant à la coalition de droite tout comme la Ligue, a jugé qu'aucun texte de loi ne permettait au conseil municipal de Bergame de prendre une telle décision et que les panneaux devaient donc être enlevés. Face à cette "attaque de la bureaucratie romaine », le responsable du parti chargé de la question linguistique, a lancé, avec la section de Bergame, un appel à manifester en défense des panneaux. On peut y lire: « les oppresseurs italiens, conscients de l'effet révolutionnaire que les panneaux bilingues et le soutien à nos cultures peuvent avoir, essayent de toutes les façons d'étouffer toute tentative destinée à donner une juste dignité à nos cultures 36 ». La section léguiste de Bergame a diffusé quant à elle un communiqué de presse accusateur daté du 2 juillet 2002 : «dans les régimes les plus durs, qu'ils soient fascistes ou communistes, dans les moments les plus sombres de l'histoire, la répression commençait toujours en incarcérant et, comme premier acte, en enlevant le nom et en donnant un numéro. Les Bergamasques ne se feront pas donner un numéro à la place du nom de leur terre : Berghèm elle était et Berghèm elle restera! ». De fait, les panneaux sont restés en place et leur situation a été régularisée en 2003 par le vote du nouveau code de la route. Leur pérennité n'était pas pour autant assurée. En effet, le nouveau maire de la ville, Roberto Bruni, élu par la coalition de gauche en 2004, a fait enlever ces panneaux peu après les élections. Il s'agissait d'ailleurs de l'un des premiers points de son programme électoral, preuve de la portée symbolique de l'enjeu toponymique.

Le conseiller communal léguiste Daniele Belotti, dans une lettre au maire, parle de la « blessure à la dignité, à l'orgueil, à la culture, à l'histoire, à la langue du peuple bergamasque » qu'a ouvert le «blitz de ferragosto 37 » avec lequel le maire a fait enlever le toponyme Berghèm ${ }^{38}$. Pour réparer cet outrage, des inconnus, dont on peut présumer sans trop de risques qu'ils étaient des militants léguistes, ont fait produire des autocollants marrons Berghèm, en tout

souvent défendu face à la Ligue, notamment quant au fait que la réforme fédérale voulue par la Ligue ne devait pas se faire aux dépens du Sud du pays (où $\mathrm{AN}$ a ses bases électorales). Cette opposition a aussi concerné la question de la Langue puisque la Ligue s'est opposée, plus ou moins ouvertement selon la conjoncture, au fait de modifier la Constitution pour y inscrire explicitement l'italien comme langue officielle (ce qui n'est toujours pas le cas aujourd'hui), alors qu'AN a porté ce projet depuis 2002. L'opposition des conseillers d'AN aux panneaux bilingues à Bergame est donc la transcription locale d'une opposition idéologique profonde qui structure la droite italienne.

36. Mailing padanismo, 3 juillet 2002.

37. Fête du 15 août, lors de laquelle les villes sont désertes. C’est le moment choisi par le maire pour enlever les panneaux «Berghèm», d'où l'accusation de Belotti qui parle de blitz.

38. L'Eco di Bergamo, 6 août 2007. Voir aussi le site de Daniele Belotti : wwww.danielebelotti.eu. Le site a aussi une version en bergamasque. 
identiques à l'ancien panneau, et les ont collés à plusieurs reprises sur la signalétique officielle en italien. En signe de protestation contre le nouveau maire de Bergame, Roberto Legramanti, maire léguiste de Cologno al Serio, une commune limitrophe, a fait rajouter aux indications routières en italien expliquant comment se rendre dans le chef-lieu de province, des indications en dialecte. Mais le maire de Bergame ne s'est pas laissé décourager par ces protestations. Non content d'avoir éliminé le Berghèm cher aux léguistes, il a fait installer en juillet 2007 dix nouveaux panneaux touristiques ornés du visage de Garibaldi et sur lesquels on peut lire : «Bergamo. Città dei Mille». Le maire voulait ainsi rendre hommage aux 180 bergamasques ayant participé à l'expédition de Garibaldi dont on fêtait le bicentenaire de la naissance. Garibaldi, héros national par antonomase, était parti en Sicile avec 1000 hommes seulement, dont presque un cinquième étaient bergamasques, afin d'unifier le pays. Les léguistes, qui considèrent que Garibaldi était venu recruter à Bergame en raison de la pauvreté de ses habitants, ont été outrés de voire ainsi la ville considérée comme le fer de lance de l'unification. Le parlementaire européen Mario Borghezio (l'un des léguistes les plus radicaux) a immédiatement réagi en menaçant : « les panneaux garibaldiens ne vont pas durer longtemps 39 ». En effet, un autocollant «Berghèm » a recouvert «Città dei Mille ». À la place du visage de Garibaldi est apparu le logo de la Ligue du Nord.

En juin 2009, un nouveau maire est élu : il s'agit de Franco Tentorio, du parti Alliance Nationale. Pour la première fois, la droite s'est présentée unie aux élections municipales. La ville est donc désormais gouvernée par Forza Italia, Alliance Nationale et la Ligue, coalition qui gouverne également le pays tout entier. Dès que la droite a repris la mairie, la question des panneaux en dialecte s'est à nouveau posée. Le maire, qui était à l'époque conseiller municipal, n'avait pas voté en 2002 en faveur des panneaux dialectaux tout comme les autres conseillers de son parti. Pourtant, devant une Ligue en pleine expansion électorale depuis les élections législatives de 2008 et désormais membre de la majorité municipale (ce qui n'était pas le cas en 2002), le maire était mal armé pour s'opposer à la requête des léguistes de remettre les panneaux en dialecte. Ainsi, le conseil communal de Bergame a approuvé en avril 2010 un ordre du jour (avec les votes favorables de la majorité de droite et défavorables de l'opposition de gauche) qui prévoit que le budget mobilise des fonds pour promouvoir des initiatives qui valorisent la «culture et la langue locale », dont la signalisation et la toponymie ${ }^{40}$. L'amendement était présenté par la Ligue et notamment par Daniele Belotti, qui avait déjà défendu les panneaux en dialecte en 2002 lors de leur installation et en 2004 lors de leur démantèlement. A ce jour (juin 2011), les panneaux «Berghèm» ne sont pas encore réapparus, mais le dernier mot de leur histoire n'a probablement pas encore été écrit. L'écriteau «Berghèm » est en revanche apparu, pour la première fois, 
en mai 2010 sur le nouveau drapeau de la province (dont Bergame est le cheflieu). Il a été voulu par le léguiste Ettore Pirovano qui préside la province depuis juin 2009 (et est aussi député) ${ }^{41}$. Certains citoyens paraissent pourtant fatigués par cette guerre toponymique et par les coûts qu'elle entraîne pour la municipalité et désormais pour la province (chaque nouveau drapeau coûtant 60 euro). C'est ainsi qu'est apparu un groupe sur Facebook intitulé «Basta cambiare $i$ cartelli "Bergamo" " (Arrêtons de changer les panneaux «Bergame») qui compte 97 membres au premier juin 2010. Mais les défenseurs de la signalétique dialectale ont aussi leur groupe sur Facebook, intitulé "Quelli che vorrebbero $i$ Cartelli dei Paesi (e Frazioni) in Dialetto » (ceux qui voudraient les panneaux des villages en dialecte) qui, lui, compte 380 membres au $1^{\mathrm{er}}$ juin 2010. La guerre toponymique qui agite le conseil municipal de Bergame depuis presque dix ans a donc trouvé sa transposition en ligne, signe qu'elle ne concerne pas que des amateurs de dialecte (généralement âgés) mais aussi les nouvelles générations qui utilisent les réseaux sociaux.

L'histoire controversée des panneaux bergamasques révèle la politisation de la question dialectale. Utilisé par la Ligue, le dialecte prend une signification xénophobe et antiunitaire, alors qu'il incarnait auparavant une forme d'expression populaire, à la limite d'une mentalité localiste, mais certainement pas un indicateur d' " anti-italianité » et encore moins d'hostilité aux étrangers. L'utilisation du dialecte est donc devenue délicate pour les maires qui ne sont pas léguistes, même si certains s'y risquent encore. Pendant l'été 2007, à Brescia, voisine de Bergame et fort semblable, sont ainsi apparues des affiches faisant la promotion de la ville. En guise de slogan, on pouvait lire «Brèsa (Brescia en dialecte). My beautiful city ». Collées partout en ville, ces affiches avaient pour but d'assurer l'autopromotion du maire de centre-gauche Paolo Corsini. La gauche peut donc encore mobiliser le dialecte. Mais, en comparaison avec Bergame, l'usage du dialecte était à Brescia d'un tout autre ordre. Les affiches de Brescia (qui sont temporaires contrairement aux panneaux qui se veulent permanents) sont ironiques. Il s'agit d'assumer l'image provinciale de la ville (incarnée par le dialecte) tout en la tournant en dérision (l'association à l'anglais et à l'image de la grande capitale). Si cette utilisation du dialecte dénote, comme à Bergame, une forme de fierté locale, elle accentue aussi son côté risible. C'est une invitation à prendre cet orgueil localiste au deuxième degré, à le relativiser, à en rire. Le rire est d'ailleurs la réaction que ces affiches ont suscitée chez les habitants de la ville que j'ai pu observer.

Le panneau signalétique disant aux visiteurs qu'ils arrivent à «Berghèm», en revanche, n'était pas là pour faire rire, mais pour faire ainsi d'une ville où l'on parle le dialecte une ville padane. Ces panneaux parlaient moins de l'amour des léguistes pour la langue locale que de leur haine de l'Italie. Leur but était également de signaler aux « extracommunautaires 42 » qu'ils ne sont 
pas chez eux. Ce n'est pas un hasard si les léguistes ont été accusés de racisme dans leurs opérations de dialectisation des panneaux, ce qui peut paraître au premier abord incongru. Le maire de Brescia n'a quant à lui jamais été l'objet de telles accusations. Dans les courriers de militants padanistes reçus par le quotidien local de Bergame, cet aspect est souvent évoqué ouvertement : ça faisait du bien de voir les panneaux en dialecte dans une ville de plus en plus « envahie par les immigrés » et risquant donc de «perdre son identité ». On comprend alors pourquoi le maire bergamasque de centre-gauche considérait ces mêmes panneaux comme « un symbole de fermeture ». S'il les a fait enlever, dit-il, c'est parce qu'il pense que les panneaux situés à l'entrée de la ville doivent s'adresser non pas aux habitants mais aux nouveaux venus qui « recherchent hospitalité et s'attendent à être accueillis 43 ».

Le cas de Cesarino Monti, maire de Lazzate (6 000 habitants, province de Milan) et sénateur léguiste, illustre parfaitement cette consubstantialité, chez les élus léguistes, de la défense du dialecte (et plus généralement de ce qu'ils appellent « l'identité ») et de la xénophobie. Cesarino Monti a ajouté «commune de Padanie » sur le panneau d'entrée de la ville, nommé «via Padania» une importante rue du centre ville, créé une rue "Soleil des Alpes » et fait installer des panneaux en dialecte dans les rues. Il a aussi voulu introduire, dans le plan d'urbanisme de 2004, l'interdiction de faire construire des édifices avec des «caractéristiques arabes » pour « ne pas dénaturer le style architectural local ». À Biassono (province de Milan), qui a aussi sa « rue Padanie », c'est le port du voile pour les femmes musulmanes que le maire léguiste a voulu interdire en 2004, condamné à une peine d'amende de 400 euros si ces femmes circulaient ainsi vêtues en ville. À Rovato (province de Brescia), en plus de baptiser une rue en hommage à la Padanie, le maire léguiste a voulu se distinguer en 2001 par une ordonnance, qu'il a dû ensuite retirer, interdisant aux « musulmans de circuler à moins de 15 mètres des églises 44 ». Pour la Ligue, donc, la « défense de l'identité » (dont le dialecte) rime avec le rejet de toute diversité religieuse, vestimentaire, voire architecturale. La désignation dialectale ou la padanisation des rues sont donc une forme de marquage du territoire dont le but est à la fois de signifier que les immigrés ne sont pas ici chez eux et que "Padania is not Italy " (autocollant produit par le parti et collé un peu partout par les militants léguistes). Ici, la toponymie dialectale sert moins à nommer (instrument de localisation), qu'à « distinguer en nommant 45 », voire à exclure en nommant.

42. Le terme " extracommunautaire", en apparence technique (il désigne officiellement les ressortissants de pays hors Communauté Européenne), est en fait méprisant. Dans le langage courant et pas seulement chez les léguistes, il désigne en effet les immigrés en provenance de pays pauvres.

43. L'Eco di Bergamo, 9 août 2007.

44. Précisons que ce type d'initiative léguiste, présentée par le parti comme une défense du catholicisme face à « l'islamisation » à laquelle on assisterait, ne suscite auprès de l'Église aucune sympathie. Bien au contraire, le Vatican a souvent critiqué le parti pour ses positions sur l'immigration, et vice versa.

45. Boyer H., art. cit., 2008, p. 10. 
La Ligue, comme le montre le cas des panneaux signalétiques, continue donc, au Nord, à monopoliser la question du dialecte. En retour, toute utilisation publique du dialecte ou toute action volontariste sont automatiquement associées au léguisme, ce qui a fortement politisé la question dialectale. A titre d'exemple, le chanteur Davide Van de Sfroos ${ }^{46}$, du fait qu'il utilise le dialecte du lac de Côme (zone où la Ligue est bien implantée), doit se justifier dans chaque interview pour ne pas apparaître comme un partisan de Bossi, et ce d'autant plus qu'il est l'idole des jeunes du parti.

En s'accaparant les dialectes, le parti, faute d'une langue, fournit à la Padanie un «patrimoine linguistique » supposé être à la base de sa spécificité. Ici donc, « la logique qui préside à l'émancipation linguistique et qui se mue en combat politique est celle de l'appropriation et de la désignation : telle langue est désignée comme appartenant intrinsèquement à telle communauté 47 ». Mais, pour la Ligue, cela signifie aussi que ceux qui ne sont pas locuteurs de la langue (ici du dialecte) sont des étrangers à la communauté, que cela concerne, notamment dans les premiers temps du léguisme, les méridionaux ou les immigrés.

Dans cette optique, l'arme toponymique se révèle particulièrement efficace : les panneaux en dialecte postés à l'entrée des villes sont pour les léguistes non seulement un signe tangible de l'extranéité de la «Padanie » vis-à-vis de l'Italie, mais aussi un avertissement disant aux étrangers qui rentrent qu'ici les padans sont "padroni a casa nostra» (" patrons chez nous », slogan sans cesse martelé par le parti).

46. Davide Van de Sfroos signifie en dialecte van di frodo, ils fraudent, en référence aux contrebandiers du Lac de Côme, où il a grandi. Son album de folk dialectal "...E Semm partii ", sorti en octobre 2001, a été vendu à plus de 50000 exemplaires.

47. Von Busekist A., "Succès et infortunes du nationalisme linguistique », in, Dieckhoff A., Jaffrelot C. (ed.), Repenser le nationalisme. Théories et pratiques, Paris, Presses de Science Po, 2006, p. 248. 